\title{
Gap junction permeability between tenocytes within tendon fascicles is suppressed by tensile loading
}

\author{
Eijiro Maeda - Shangjun Ye · Wen Wang • \\ Dan L. Bader • Martin M. Knight • David A. Lee
}

Received: 4 January 2011 / Accepted: 9 June 2011 / Published online: 25 June 2011

(C) The Author(s) 2011. This article is published with open access at Springerlink.com

\begin{abstract}
Gap junction communication is an essential component in the mechanosensitive response of tenocytes. However, little is known about direct mechanoregulation of gap junction turnover and permeability. The present study tests the hypothesis that mechanical loading alters gap junction communication between tenocyte within tendon fascicles. Viable tenocytes within rat tail tendon fasicles were labelled with calcein-AM and subjected to a fluorescent loss induced by photobleaching (FLIP) protocol. A designated target cell within a row of tenocytes was continuously photobleached at $100 \%$ laser power whilst recording the fluorescent intensity of neighbouring cells. A mathematical compartment model was developed to estimate the intercellular communication between tenocytes based upon the experimental FLIP data. This produced a permeability parameter, $k$, which quantifies the degree of functioning gap functions between cells as confirmed by the complete inhibition of FLIP by the inhibitor $18 \alpha$-glycyrrhentic acid. The application of $1 \mathrm{~N}$ static tensile load for $10 \mathrm{~min}$ had no effect on gap junction communication. However, when loading was increased to $1 \mathrm{~h}$, there was a statistically significant reduction in gap junction permeability. This
\end{abstract}

Electronic supplementary material The online version of this article (doi:10.1007/s10237-011-0323-1) contains supplementary material, which is available to authorized users.

E. Maeda $\cdot$ S. Ye $\cdot$ W. Wang · D. L. Bader · M. M. Knight $(\bowtie)$.

D. A. Lee

School of Engineering and Materials Science,

Queen Mary, University of London, Mile End Road,

London E1 4NS, UK

e-mail: M.M.Knight@qmul.ac.uk

S. Ye

State Key Laboratory of Fluid Power Transmission and Control,

Zhejiang University, Hangzhou, China coincided with suppression of connexin 43 protein expression in loaded samples as determined by confocal immunofluorescence. However, there was an upregulation of connexin 43 mRNA. These findings demonstrate that tenocytes remodel their gap junctions in response to alterations in mechanical loading with a complex mechanosensitive mechanism of breakdown and remodelling. This is therefore the first study to show that tenocyte gap junctions are not only important in transmitting mechanically activated signals but that mechanical loading directly regulates gap junction permeability.

Keywords Tendon · Gap junction · FLIP · Connexin · Mechanotransduction

\section{Introduction}

Tendon is a dense connective tissue, mostly consisting of collagen fibres running parallel to the longitudinal axis of its structure and the main axis of tensile loading (Kannus 2000). Aligned along the collagen fibres are tendon-specific cells, tenocytes, which are interconnected both longitudinally and laterally via gap junctions (McNeilly et al. 1996). As tendon transmits mechanical force generated by muscle, it is repeatedly subjected to mechanical loading that has been demonstrated to influence tenocyte metabolism. For example, the application of mechanical loading within a physiological range produces anti-catabolic (Lavagnino et al. 2003; Asundi and Rempel 2008; Maeda et al. 2009, 2010) and anabolic effects (Banes et al. 1999b; Maeda et al. 2007), while catabolic responses are enhanced when the mechanical loading falls outside the physiological range (Flick et al. 2006; Leigh et al. 2008). The associated mechanically induced signalling is transmitted between tenocytes via intercellular gap junction communication (Banes et al. 1999b). 
Gap junctions consist of two hemi-channels, termed connexons, an aggregate of six connexin protein subunits, with a central pore. In the open state, small species $(<1 \mathrm{kDa})$, such as metabolites and ions, are able to pass freely through the gap junctions (Goldberg et al. 1999). By transporting these molecules and ions to neighbouring cells, tendon cells communicate with each other to produce a coordinated response to a variety of stimuli, including mechanical perturbation. Tenocytes within tendon fascicles express a variety of connexin sub-types, most notably connexins 26, 32 and 43 (McNeilly et al. 1996; Maeda et al. 2010; Wagett et al. 2006). Connexin 43 is localised predominantly within gap junctions between cells in rows oriented along the collagen fibres. By contrast, connexins 26 and 32 have a more diffuse localisation pattern, possibly forming gap junctions both longitudinally and laterally between adjacent cell rows (McNeilly et al. 1996). Connexin 43 gap junctions have been shown to mediate the inhibition of collagen synthesis in tenocytes in response to mechanical loading, whereas those involving connexin 32 may have a stimulatory role (Wagett et al. 1996). Indeed, non-specific inhibition of gap junctions has been shown to suppress the stimulatory effects of mechanical loading on DNA and collagen synthesis (Banes et al. 1999b).

Gap junctions are highly dynamic structures, and their communication can be influenced by events both transient (seconds) and longer term (minutes to hours), permitting the precise regulation of their permeability/conductance and ultimately functionality. For example, rapid events, involving alterations in transjunctional voltage, $\mathrm{pH}$, phosphorylation or intracellular calcium levels, can affect the gating status of gap junctions, in effect regulating the opening and closing of the central pore. In addition, gap junctions exhibit rapid turnover rates, with half-lives reported to be $1-5 \mathrm{~h}$ in cultured cells and within intact tissue (Segretain and Falk 2004). Thus, the regulation of gap junction assembly/disassembly, turnover and trafficking to the plasma membrane may also play a key role in the control of intracellular communication. However, data reporting the effects of mechanical perturbation of these processes and the fundamental gap junction functionality are limited. Direct effects of mechanical loading on gap junction formation and intercellular communications have been reported in vascular endothelial cells (DePaola et al. 1999). For isolated tenocytes, discontinuous cyclic 5\% tensile strain at $1 \mathrm{~Hz}$ induces alterations in connexin 43 protein expression compared with unstrained cells (Banes et al. 1999a). Although these studies suggest that gap junction expression in tenocytes is mechanosensitive, the permeability of gap junctions between tenocytes within intact tendon fascicles has not been investigated, neither has the direct regulation of gap junctional communications by mechanical stimulation.

In the present study, we test the hypothesis that mechanical loading alters gap junctional communications between tenocytes within tendon fascicles. Studies investigating gap junction permeability within isolated cells or within intact tissues typically utilise techniques based on the transport of permeable dyes, associated with advanced live cell imaging techniques. In the current study, a fluorescence loss induced by photobleaching (FLIP) technique has been developed in conjunction with confocal laser microscopy. The experimental technique is complemented by a mathematical approach involving a compartment model to characterise and quantify gap junctional communications in tenocytes in situ with or without mechanical perturbation.

\section{Methods}

\subsection{Tendon fascicle samples}

Fascicles were dissected from the tail tendon of male Wistar rats (4-5 months old), under aseptic conditions. Both the tendon and isolated fascicles were kept moistened with Dulbecco's modified Eagle's medium (DMEM) supplemented with $10 \%$ foetal calf serum (FCS), $150 \mathrm{mgl}^{-1}$ ascorbic acid, $0.02 \mathrm{M}$ L-glutamine, $1 \%$ non-essential amino acids, $0.01 \mathrm{M}$ Hepes, $50 \mathrm{U} \mathrm{ml}^{-1}$ penicillin and $0.05 \mathrm{mg} \mathrm{ml}^{-1}$ streptomycin (DMEM + 10\%FCS, all from Sigma-Aldrich, Poole, UK). Dissected fascicles were pooled in a culture dish filled with DMEM $+10 \%$ FCS and examined on the day of dissection.

\subsection{Experimental FLIP analysis of intercellular communication}

A gap junction permeable fluorescent tracer, calcein $(623 \mathrm{Da})$, was introduced into the tenocytes by incubation of the fascicles in DMEM $+10 \%$ FCS $+10 \mu \mathrm{M}$ Calcein-AM (Invitrogen) for $1 \mathrm{~h}$ at $37^{\circ} \mathrm{C}$. Each sample was washed three times in DMEM+10\% FCS and mounted on a custommade rig, which enables visualization of cells during confocal imaging while maintaining the fascicles at $37^{\circ} \mathrm{C}$ in $\mathrm{DMEM}+10 \% \mathrm{FCS}$. Fluorescence loss induced by photobleaching (FLIP) was used to evaluate gap junction communication, using a confocal laser microscope (TCS SP2, Leica Microsystems) and a $\times 40$ oil immersion objective lens. The confocal pin hole diameter was $82 \mu \mathrm{m}$ with a beam expander setting of 6 . Confocal images $(27 \times 220 \mu \mathrm{M})$ were obtained from a row of at least 4 individual tenocytes, at a depth of 10-30 $\mu \mathrm{M}$ from the fascicle surface (Fig. 1a), with a defined target cell subjected to repetitive photobleaching using the fully automated Leica software. The FLIP protocol consisted of a series of 140 consecutive images made during a 420-s imaging period, such that the pixel scanning time for each image was $3 \mathrm{~s}$ (Fig. 1b). Laser excitation was provided at $488 \mathrm{~nm}$ and $3 \%$ full power with emission detected above $500 \mathrm{~nm}$. Laser power was set to automatically increase to 
(a)

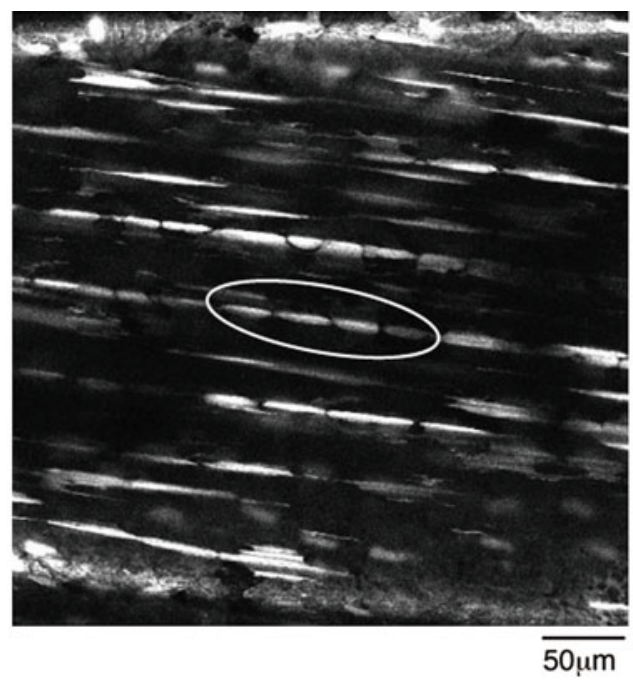

(b)

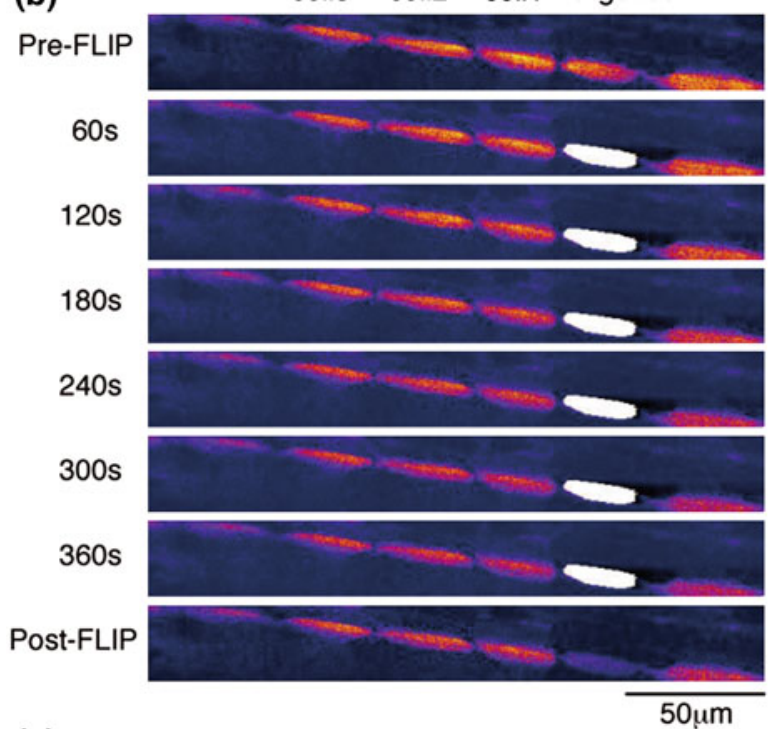

(c)

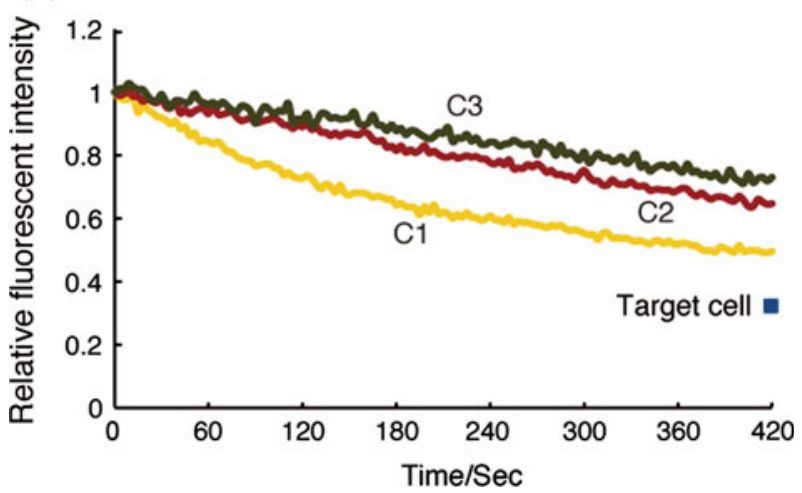

Fig. 1 The fluorescence loss induced by photobleaching (FLIP) technique was successfully used on tenocytes in tendon fascile. Representative images and data from the FLIP protocol for tenocytes labelled with Calcein-AM. a Low-magnification confocal image showing the location of a group of cells suitable for FLIP analysis. b Selected confocal images from a representative FLIP protocol and $\mathbf{c}$ the resulting fluorescent decay profiles for cells 1, 2 and $3(\mathrm{C} 1, \mathrm{C} 2$ and $\mathrm{C} 3)$ as well as the post-FLIP intensity in the target cell (a)
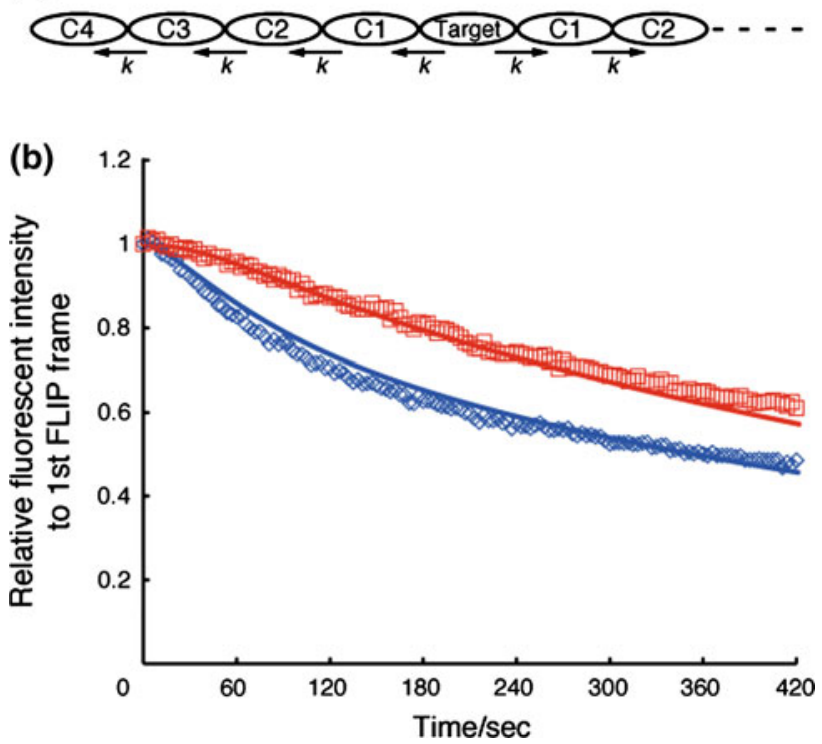

Fig. 2 The mathematical compartment model accurately described the experimental FLIP data. a Schematic representation of the compartment model for the transfer of bleached and non-bleached molecules during the FLIP protocol. b Representative data showing the changes in fluorescence during the FLIP protocol for cell $1(\mathrm{C} 1$, blue $)$ and cell $2(\mathrm{C} 2$, $r e d)$ and the close agreement between the model predictions (lines) and experimental data (points). For this individual data set, the $k$ value was 0.0206

$100 \%$ full power when scanning the target cell, defined by a region of interest, in each consecutive image. Individual images of each group of cells were also obtained pre- and post-FLIP using 3\% laser power.

For each image in the FLIP sequence, the mean fluorescence intensity was calculated in the 3 cells adjacent to the bleached target cell. These were designated cell 1,2 and 3 (C1, C2 and C3) as shown in Figs. 1b and 2a. The cellular intensity values were normalised to the values measured in the same cells prior to the start of the FLIP protocol. Temporal changes in the relative intensity were used for subsequent mathematical analysis described below. The relative intensities at the end of the FLIP protocol for C1-3, as well as the target cell, were calculated from the pre- and post-FLIP images and used for statistical analysis.

Non-photobleached control experiments were carried out by scanning the entire image at $3 \%$ full power laser to confirm that this induced minimal photobleaching. To examine whether the fluorescent decay in neighbouring cells was a direct result of intercellular transfer of bleached calcein tracer molecules via gap junctions, stained fascicles were further incubated in DMEM $+10 \%$ FCS with the presence of a gap junction antagonist, $18 \alpha$-glycyrrhentic acid $(18 \alpha-$ GA, Sigma-Aldrich) at $100 \mu \mathrm{M}$ for $1 \mathrm{~h}$ at $37^{\circ} \mathrm{C}$. Fascicles were then subjected to the FLIP protocol in the presence of $18 \alpha$-GA. One-way analysis of variance was performed to 
assess the differences between the non-photobleached control, inhibitor and normal FLIP groups, followed by Tukey's multiple comparison test, with the significance level set at $p<0.05$.

\subsection{Mechanical loading of tendon fasicles for FLIP study}

To test the hypothesis that gap junction communication is regulated by mechanical loading, the FLIP protocol was performed following a period of static loading at $1 \mathrm{~N}$ (Loaded group). FLIP was performed in two different locations in each fascicle. Control experiments were carried out in an identical manner, but with the fascicle maintained in an unloaded state in the rig (Unloaded group). The custom-made loading rig mounts on the inverted stage of the confocal microscope and is similar to that previously described (Screen et al. 2004). The fascicle specimens were cut to provide a $20 \mathrm{~mm}$ grip-to-grip length with an additional $2 \mathrm{~mm}$ at either end for gripping. Static loading of approximately $1 \mathrm{~N}$ was applied by hanging a $100 \mathrm{~g}$ mass connected to the fascicle grip via a pulley. A load cell was connected to the grip at the other end of the fascicle. Loading was applied instantaneously, and FLIP imaging was conducted after either $10 \mathrm{~min}$ or $1 \mathrm{~h}$ of static loading by which time any creep displacement was minimal. Fascicles were maintained at $37^{\circ} \mathrm{C}$ in DMEM $+10 \%$ FCS. The difference in gap junction permeability between the two groups was assessed from temporal fluorescence decay profiles, using a mathematical model as described in the following section.

\subsection{Theoretical modelling of intercellular communication}

The transport of fluorescent tracer molecules between adjacent cells was described using a compartment model. The model was based on the assumption that intercellular transport of the calcein tracer molecule occurs exclusively through direct cell-cell communication with no dye leakage across the cell membrane. Furthermore, it is assumed that the diffusive intercellular transport between cells is considerably slower than the intracellular transport within a cell. Thus, the concentration of the bleached tracer molecule in the $i$ th cell $c_{i}$ can be defined as the amount of the bleached molecule per unit volume, i.e., $c_{i}=N_{i} / V_{i}$, where $N_{i}$, and $V_{i}$ are the amount of the bleached molecules and the volume in the $i$ th cell, respectively. The change of $N_{i}$ is assumed to follow the mass conservation equation (1), where $A_{i, j}$ and $P_{i, j}$ are the effective contact area and the area-averaged permeability, respectively, between the $i$ th and the $j$ th cells.

$$
\begin{aligned}
\frac{\mathrm{d} N_{i}}{\mathrm{~d} t}= & p_{i-1, i} A_{i-1, i}\left(\frac{N_{i-1}}{V_{i-1}}-\frac{N_{i}}{V_{i}}\right) \\
& -p_{i, i+1} A_{i, i+1}\left(\frac{N_{i}}{V_{i}}-\frac{N_{i+1}}{V_{i+1}}\right)
\end{aligned}
$$

This set of equations was solved with appropriate boundary and initial conditions (details can be found in Supplementary material).

In the present experiment, the FLIP protocol consists of a series of 140 consecutive steps in each experiment, which lasted $420 \mathrm{~s}$. In each step, the target cell, i.e., C0, is scanned at full power for less than $0.2 \mathrm{~s}$, resulting in a percentage of the unbleached molecules, $\alpha$, being bleached,

$\bar{N}_{0}=N_{0}^{*}+\alpha\left(N_{\text {total }}-N_{0}^{*}\right)$

where $N_{0}^{*}$ and $\bar{N}_{0}$ are the amount of the bleached molecule before and after each step, and $N_{\text {total }}$ is the total amount of the molecule in the target cell, which remains unchanged. All cells are assumed to have identical properties in the model, so that only cells adjacent to one side of the target cell were analysed, as indicated in Fig. 2a. In all experiments, no significant intensity change was observed in the fourth cell furthest from the target cell. Thus, the compartment model, developed to mimic the intercellular transport of bleached molecules, was restricted to the target cell and 4 cells on either side. Thus, the index $i$ is $0-4$, and the amount of bleached molecule in the fifth compartment, $N_{5}$, is set to zero. Symmetrical boundary conditions are implemented for the $\mathrm{C} 0$ compartment such that transport occurs equally to both sides of the target cell. Initial amount of bleached molecule is assumed to be zero in all compartments. Details of the model are provided in the Supplementary material, where analytical solutions for the amount of bleached molecules in all the compartments are achieved and can be expressed in an array form as given in (3), where $N(t)=\left(N_{0}(t), \ldots, N_{4}(t)\right) . \lambda_{i}$ and $u_{i}$ are the eigenvalue and eigenvector of the coefficient matrix. $\bar{N}$ is the array form of the initial value, and $k=P A / V$ is a specific parameter for the intercellular molecular permeability, where $P, A$ are the identical values of intercellular permeability, contact area and cell volume, respectively.

$N(t)=\sum_{i=0}^{4} \mathbf{u}_{\mathrm{i}} \mathrm{e}^{\lambda_{i} k t} \mathbf{u}_{i}^{*} \bar{N}$

Estimated fluorescent decay profiles for $\mathrm{C} 1$ and $\mathrm{C} 2$ were fitted to experimental data, and $k$ which gave the least squared difference between the model estimation and the experimental values was determined. Due to the non-normal distribution of the data, Wilcoxon rank sum tests were used to assess the difference in $k$ between loaded and unloaded samples with the significance level set at $p<0.05$.

\subsection{Gene expression analysis}

In a separate set of experiments, static loading of $1 \mathrm{~N}$ was applied to fascicles for $1 \mathrm{~h}$ at $37^{\circ} \mathrm{C}, 5 \% \mathrm{CO}_{2}$ using a previously characterised incubator-mounted loading system (Maeda et al. 2009). Control samples remained unloaded. 
Table 1 Primer sequences of mRNA for connexin $(\mathrm{Cx})$ 26, 32 and 43

\begin{tabular}{llccc}
\hline Gene & Sequence & Position & Length & Concentration (nM) \\
\hline Cx26 & & & 95 & \\
Forward & AGCAAAGATGAGGGAGAGGATGAG & 977 & & 300 \\
$\quad$ Reverse & CGGCATTTAAGGTGGGAATGTTTG & 1,071 & & 300 \\
Cx32 & & & 87 & 200 \\
$\quad$ Forward & AGACACGCCTGCATACAT & 6 & & 200 \\
$\quad$ Reverse & AGTTCATCCTGCCTCATTC & 92 & 91 & 300 \\
Cx43 & & & & 300 \\
Forward & CTCCACTCTCGCCTATGTC & 1,023 & & \\
Reverse & CTAGCTTGCTTGTTGTAATTGC & 1,113 & & \\
\hline
\end{tabular}

Both loaded and unloaded fascicles were collected at the end of the incubation period, washed briefly in RNase and DNase-free phosphate-buffered solution (Cambrex, NJ, USA), frozen in liquid nitrogen and stored at $-80^{\circ} \mathrm{C}$.

Total RNA was extracted from the fascicles using Trireagent (Sigma-Aldrich). The RNA was purified with RNeasy mini-columns and reagents (RNeasy Mini Kit, Qiagen, Crawley, UK) in accordance with the protocol provided by the manufacturer, in conjunction with a RNase-free DNase I digestion kit (Qiagen) to remove genomic DNA from the preparation. cDNA was synthesised from $100 \mathrm{ng}$ of the total RNA using a commercially available kit (AffinityScript QPCR cDNA synthesis kit, Agilent Technologies UK, Cheshire, UK). The Co-RT method (Zhu and Altmann 2005) was adopted to transcribe both mRNA and 18s rRNA (18S) after modifications; the latter was transcribed with the random primer provided in the kit.

The expression levels of three connexin genes, connexins 26, 32 and 43 (Cx26, Cx32 and Cx43, respectively), were selected for quantitative PCR assessment (qPCR). Primers were designed and purchased from a commercial source (Sigma-Genosys, Poole, UK) (Table 1). For the amplification of 18S, QuantumRNA Universal 18S Internal Standard kit (Ambion, Applied Biosystems, Warrington, UK) was used. Each cDNA was assessed in duplicate for each of a total of four genes, using Brilliant II SYBR Green QPCR Master Mix (Agilent Technologies UK). qPCR was performed with a thermal cycler (Mx3000P, Stratagene, CA, USA) according to the prescribed protocol. This introduced an initial heat activation at $95^{\circ} \mathrm{C}$ for $10 \mathrm{~min}, 40$ cycles of $95^{\circ} \mathrm{C}$ for $30 \mathrm{~s}$ and $60^{\circ} \mathrm{C}$ for $60 \mathrm{~s}$, followed by a dissociation programme.

The relative expression level of each connexin mRNA, normalised to $18 \mathrm{~S}$, was determined in the loaded and unloaded samples as described previously (Maeda et al. 2009). Subsequently, the expression ratios of loaded samples to unloaded samples were determined, with paired comparison between fascicles dissected from the same tendon. Paired t-tests were performed to assess the difference in $\log _{2}$ relative expression level between loaded and unloaded groups, with the significance level set at $p<0.05$.

\subsection{Confocal immunofluorescence}

The localisation of connexins 26, 32 and 43 within the tendon fascicle was assessed via a standard indirect immunofluorescence technique. Selected loaded and unloaded fascicles were snap-frozen in liquid nitrogen-cooled isopentane (Sigma-Aldrich). The frozen tissues were embedded in Tissue-Tek OCT compound, and sections, with a thickness of 8-12 $\mu \mathrm{M}$, were cut, transferred onto glass slides, and stored at $-80^{\circ} \mathrm{C}$ prior to immunolocalisation. The sections were fixed in $100 \%$ methanol at $-20^{\circ} \mathrm{C}$ overnight and blocked by incubation in PBS containing $0.1 \%(\mathrm{w} / \mathrm{w}$ ) bovine serum albumin (BSA, Sigma-Aldrich) for $5 \mathrm{~min}$ three times, followed by incubation in PBS containing 5\% goat serum (SigmaAldrich) and three further washes in PBS containing $0.1 \%$ BSA. The sections were incubated in one of the following primary antibodies diluted in PBS containing $0.1 \%$ BSA at $37^{\circ} \mathrm{C}$ for $1 \mathrm{~h}$ : rabbit anti-Cx26 IgG $(10 \mu \mathrm{g} / \mathrm{ml})$, rabbit antiCx32 IgG $(2 \mu \mathrm{g} / \mathrm{ml})$, and rabbit anti-Cx43 IgG $(2 \mu \mathrm{g} / \mathrm{ml})$ (all polyclonal, Invitrogen). Appropriate controls were included. After three washes, the sections were incubated in Alexa Flour555-conjugated anti-rabbit (1:400, Invitogen) at $37^{\circ} \mathrm{C}$ for $1 \mathrm{~h}$. The samples were mounted and counterstained with DAPI, for visualization of tenocyte nuclei (ProLong with DAPI Invitrogen). Fluorescence images were taken using a confocal laser microscope (TCS SP2, Leica).

\section{Results}

Continuous photobleaching of the target cell induced by the FLIP protocol resulted in a rapid and significant decrease in the fluorescent intensity in the immediate neighbouring cell, C1 (Fig. 1). By contrast, there appeared to be a slight delay in the initiation of the fluorescent decay in $\mathrm{C} 2$ and a further delay in $\mathrm{C} 3$, with a smaller overall decrease in the intensity in these cells. The compartment model, developed for the FLIP protocol, generated characteristic temporal intensity decay profiles for $\mathrm{C} 1$ and $\mathrm{C} 2$. Indeed, there was good agreement between model predictions and experimental results (Fig. 2b). Nonphotobleached control FLIP experiments demonstrated that 
(a) FLIP profiles

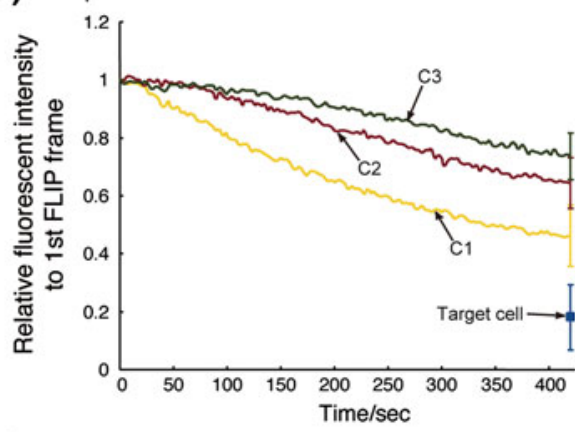

(b) Non-photobleached control

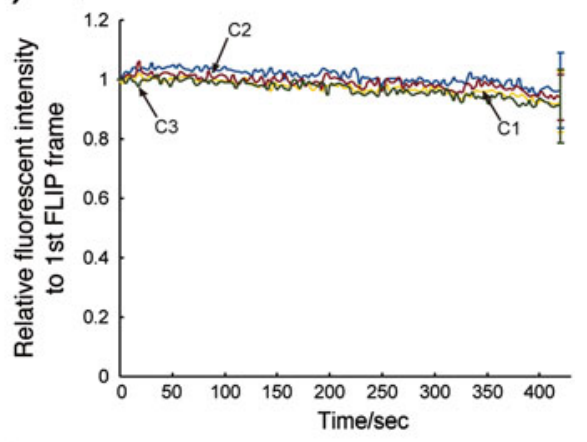

(c) FLIP with the presence of $18 \alpha-$ GA

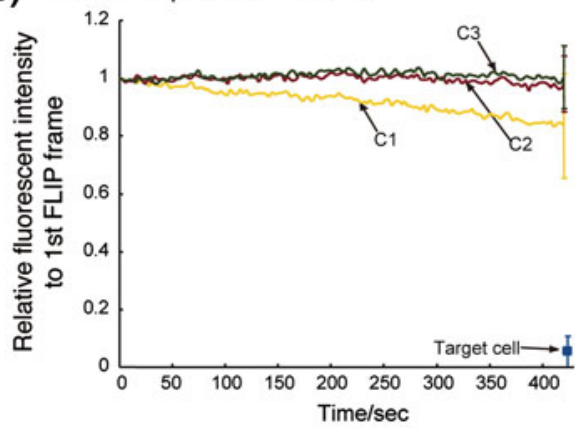

(d) End point intensity

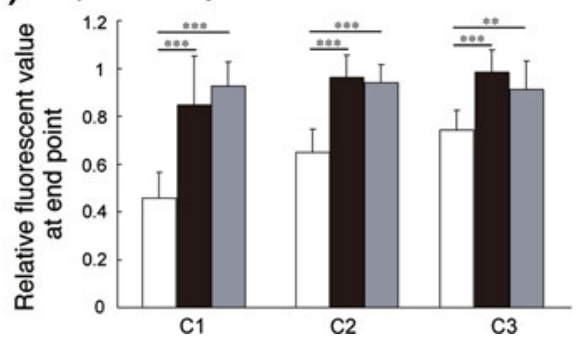

Fig. 3 FLIP is caused by intercellular communication via gap junctions. a Mean normalised fluorescent intensity profiles of cell 1 (C1), cell 2 (C2) and cell 3 (C3) as well as the post-FLIP intensity of target cell (blue) based upon FLIP experiments on untreated, unloaded fascicles. b Mean normalised fluorescent intensity profiles from non-photobleached control FLIP experiments without specific photobleaching of a target cell. $\mathbf{c}$ Mean normalised fluorescent intensity profiles from FLIP experiments conducted on fascicles treated with the gap junction blocker $18 \alpha$-glycyrrhentic acid ( $18 \alpha$-GA). d End point values after $420 \mathrm{~s}$ for $\mathrm{C} 1$, $\mathrm{C} 2$ and C 3 for non-treated samples (white bar $n=10$ ), inhibitor-treated samples (black bar $n=11$ ) and non-photobleached control samples (grey bar $n=12$ ). Statistically significant differences are indicated at $p<0.05$ (*) $^{\circ} p<0.01(* *)$ and $p<0.001(* * *)$

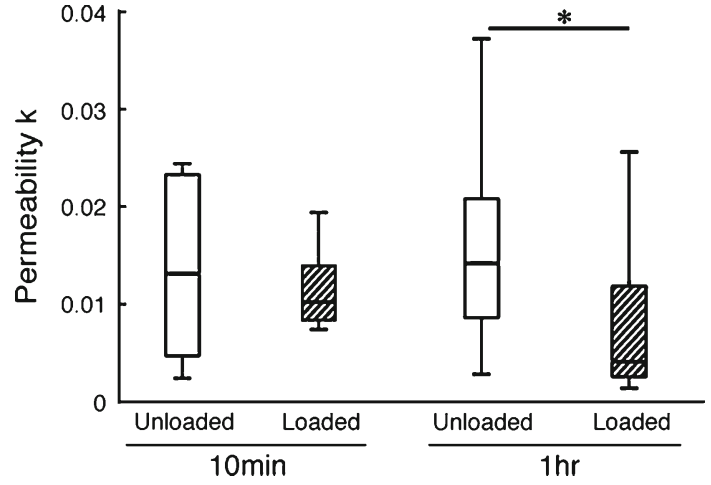

Fig. 4 Mechanical loading for $1 \mathrm{~h}$ reduces gap junction permeability. Permeability parameter $k$ (units: $\mathrm{s}^{-1}$ ) for intercellular gap junction communication in unloaded fascicles and those subjected to static $1 \mathrm{~N}$ loading for $10 \mathrm{~min}$ and $1 \mathrm{~h}$. Statistically significant differences are indicated at $p<0.05(*) . n=7,8,10$ and 14

there was minimal decay in the fluorescent intensity, indicating that scanning with $3 \%$ of full power did not contribute to the observed decrease fluorescent intensities within the FLIP experiment (Fig. 3b). Moreover, when fascicles were treated with the gap junction inhibitor, $18 \alpha$-GA, there was no temporal decay in fluorescent intensity in cells 1,2 and 3 (Fig. 3c). By contrast, there was a dramatic photobleaching of the target cell, attaining an end point relative intensity of 0.05 compared with a value of 0.2 for the target cell in the absence of $18 \alpha$-GA (Fig. 3a, c). Pooled data also indicated statistically significant reductions in the end point values for $\mathrm{C} 1, \mathrm{C} 2$ and $\mathrm{C} 3$ between non-treated controls and the inhibitor-treated group (Fig. 3d). There were no differences between corresponding values for the inhibitor-treated and non-photobleached control groups.

The compartment model fitting to the experimental FLIP data obtained from cells in 10 control fascicles (Fig. 3a) produced a median value for the permeability parameter $k$, of $0.0124 \mathrm{~s}^{-1}$, which is equivalent to an absolute permeability value of $0.248 \mu \mathrm{M} / \mathrm{s}$ based on an approximated tenocyte length of $20 \mu \mathrm{M}$. This estimated absolute permeability between tenocytes is similar to the value of $0.128 \mu \mathrm{M} / \mathrm{s}$ estimated in a previous model (Eckert 2006) (see "Supplementary material" for more details). Mean photobleaching efficiency, $\alpha$, was estimated to be $5.0 \%$, which is close to the value of $9.6 \%$ obtained in previous studies where fluorescent intensity of a single cell was measured directly after photobleaching.

The application of static loading for $10 \mathrm{~min}$ produced no significant differences in the permeability value between loaded and unloaded samples (Fig. 4). By contrast, extending the static loading to $1 \mathrm{~h}$ inhibited the gap junction communications, characterised by a statistically significant reduction in the intercellular permeability for loaded samples compared with unloaded controls (Fig. 4). 

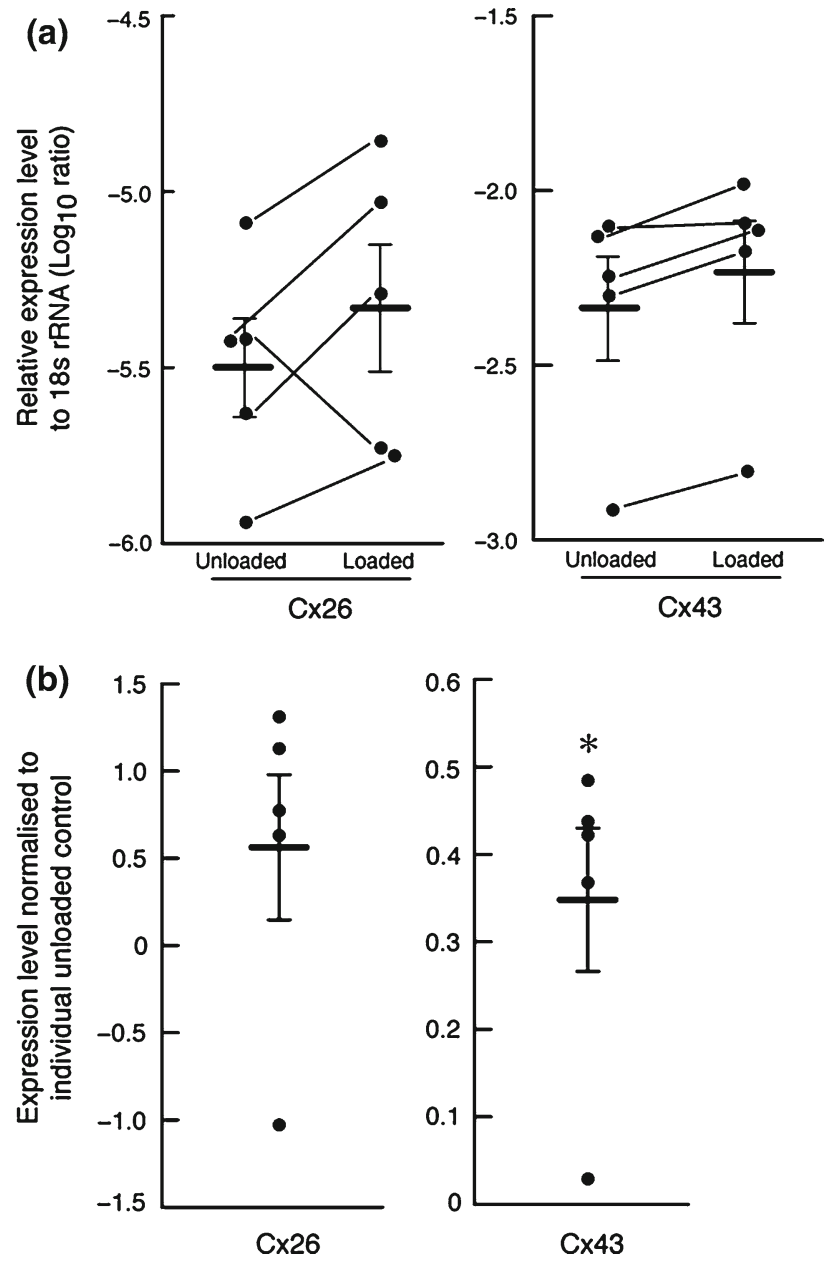

Fig. 5 Connexin 43 gene expression is up-regulated following $1 \mathrm{~h}$ of mechanical loading. a Relative expression levels of Cx26 and Cx43 for unloaded and loaded tendon fascicles. Data have been normalized to $18 \mathrm{~S}$ rRNA and plotted on a $\log _{10}$ scale. b Relative expression ratio of each loaded sample to their corresponding unloaded control on $\log _{2}$ scale $(0$ represents the unloaded control level). Horizontal bars represent mean ratio of each gene \pm the standard error. Statistically significant differences are indicated at $p<0.05(*)$

Messenger RNA expression for the three connexin genes in the rat tail tendon specimens, measured at the end of $1 \mathrm{~h}$ static loading period, demonstrated robust expression of both Cx43 and Cx26 (Fig. 5a). By contrast, Cx32 expression was at or below the limit of detection; in over $40 \%$ of samples, fluorescent intensity did not exceed the background threshold during 40 PCR cycles, with the remainder typically exhibiting $\mathrm{Ct}$ values between 35 and 40. Accordingly, further analysis of Cx32 expression was not attempted and subsequent comparison between loaded and unloaded samples focused on $\mathrm{Cx} 26$ and $\mathrm{Cx} 43$. A significantly increase in expression levels for $\mathrm{Cx} 43$ was observed in loaded samples when compared to unloaded controls (Fig. 5b). By contrast, no significant differences were exhibited for Cx26 expression between the loaded and control samples. Connexin 26 protein expression
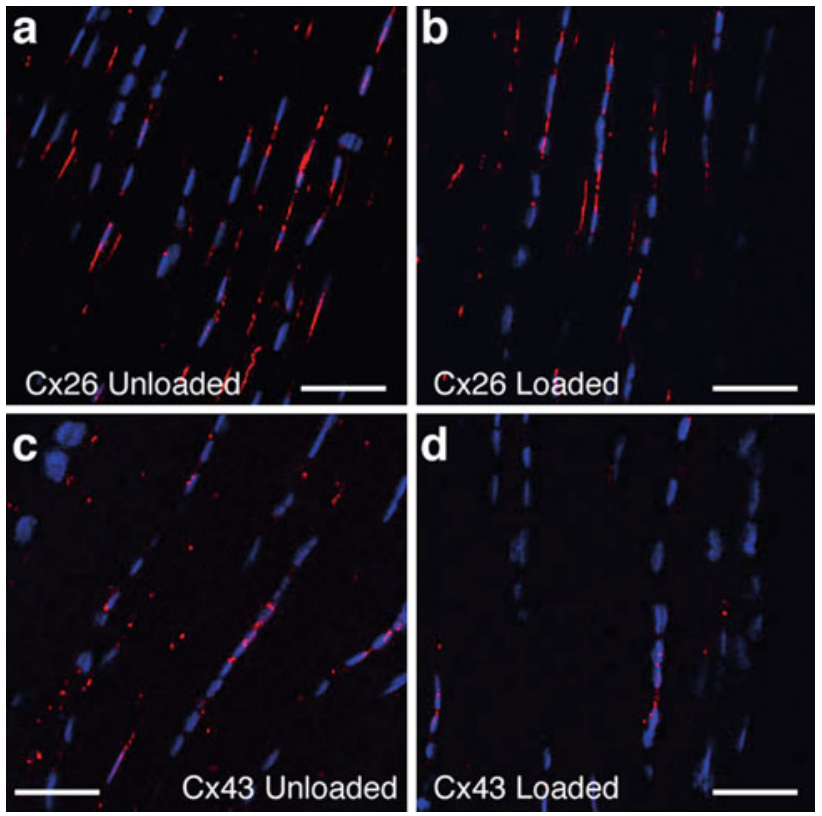

Fig. 6 Connexin 43 protein expression is up-regulated following $1 \mathrm{~h}$ of mechanical loading. Confocal images of immunolabelled connexins in isolated tendon fascicles. Cx26 in unloaded (a) and loaded fascicles (b); Cx43 in unloaded (c) and loaded fascicles (d). Scale bar indicates $50 \mu \mathrm{M}$

was confirmed by immunofluorescence in both unloaded and loaded fascicles (Fig. 6a, b). Connexin 26 localisation was predominantly cytoplasmic and diffuse in nature and was not altered by the application of $1 \mathrm{~h}$ static loading of $1 \mathrm{~N}$ (Fig. 6b). By contrast, connexin 43 was clearly localised discretely at the contacting point of neighbouring cells in the same row within the unloaded samples (Fig. 6c). In the loaded fascicles, connexin 43 protein localization was markedly altered with a loss of the clear discrete staining between cells evident in the unloaded fascicles and an overall reduction in staining (Fig. 6d). Connexin 32 was not observed in either loaded or unloaded rat tail tendon fascicles using immunolocalisation, (data not shown), in agreement with gene expression data.

\section{Discussion}

Mechanical stimulation has been demonstrated to be a key regulatory factor of tenocyte metabolism and subsequent tendon tissue homeostasis in terms of both biochemical and biomechanical integrity (Wang 2006). Previous studies have investigated early events of the mechanotransduction process of tenocytes, suggesting that single tenocytes are mechanoresponsive and also that gap junction communication between tenocytes plays a role in mechanotransduction through the transmission of mechanically activated signalling (Wall and Banes 2005). However, to our knowledge, this is the first study to address the mechanoregulation of gap junction com- 
munications within intact tendon fascicles by direct assessment of gap junction communication in the loaded and unloaded states.

The FLIP technique was adopted to monitor gap junction communications between tenocytes within a tissue explant. Other confocal laser microscopy techniques, such as fluorescent recovery after photobleaching (FRAP), have also been used to analyse gap junction communications (Abbaci et al. 2007), but are limited to investigation of the target cell and its immediate neighbour. By contrast, FLIP provides the potential to analyse multiple cell-cell communications in isolation. An alternative approach involves the selective introduction of a fluorescent dye to a single cell, for example via microinjection, with subsequent observation of the spread of the dye to neighbouring cells. This is impractical via microinjection for cells in situ within the tissue, but may be possible using photo-uncaging fluorophores. However, FLIP does not require the incorporation of the dye into a single target cell as this technique traces the transport of a bleached tracer through adjacent cells. Using this FLIP technique, the present study quantifies the permeability of intercellular communication between adjacent tenocytes. The complete inhibition of FLIP by $18 \alpha$-GA confirms that this dye transfer is mediated by gap junction communication. The non-specific nature of $18 \alpha$-GA inhibition is such that it does not selectively target gap junctions involving a specific connexin type. However, the current results indicate a higher level of gene expression of Cx43, when compared to Cx 26 and 32 (Fig. 5a). In addition, connexin 43 was preferentially localised to the interspace between adjacent cells, whereas connexin 26 showed a more cytoplasmic distribution (Fig. 6). Thus, connexin 43 appears a likely candidate for the predominant gap junction protein involved and is also reported to exhibit mechanosensitive expression in other cell types (DePaola et al. 1999; Garcia and Knight 2010). To date, only few theoretical models have been reported to provide further analysis of FLIP data (Pan et al. 2009). Accordingly, a 2-dimensional compartment model of the FLIP protocol was developed to derive a parameter relating to intercellular permeability $k$, independent of specific imaging protocols. Previous studies have reported that tenocytes form gap junctions not only between cells arranged within a single row but also between cells in separate rows (McNeilly et al. 1996). However, the current compartment model was restricted to longitudinal intercellular communication. Nevertheless, the fact that the model matches closely with experimental FLIP data (Fig. 2b) suggests that the major communication is between cells within rows, as opposed to between rows.

The current study examined the effect of a $1 \mathrm{~N}$ static load which is equivalent to applying $4-5 \mathrm{MPa}$ of tensile stress, based on the measurement of the fascicle diameter postloading. This value corresponds to approximately $10 \%$ of tensile strength of rat tail tendon fascicle, resulting in a tensile strain of approximately 5\% (Screen et al. 2004). Static loading has a well-known catabolic effect on tendon and other connective tissues. However, in previous studies of the effects of mechanical loading on tenocyte metabolism, we have demonstrated that short-term application of 5\% cyclic tensile strain also induces a catabolic response by the tenocytes (Maeda et al. 2009, 2010). Previous studies suggest that lower levels of tensile stress, less than approximately 2.5 MPa, have broadly anabolic (Abreu et al. 2008; Yamamoto et al. 2002) and anti-catabolic (Marsolais et al. 2007; Arnoczky et al. 2004) effects on tenocytes. Accordingly the static mechanical loading regime employed in this study is likely to be catabolic in nature, at least in the short term. However, numerous studies indicate a complex temporal response to mechanical loading where by short-term catabolic effects are followed by an anabolic recovery (e.g. Maeda et al. 2009). Furthermore, whilst the response to more anabolic cyclic loading would certainly be interesting, this presents methodological difficulties in allowing simultaneous confocal imaging.

The application of a $1 \mathrm{~N}$ load for $1 \mathrm{~h}$ resulted in a reduction in intercellular communication. This was characterised by a statistically significant reduction in the permeability parameter $k$, with median values of $0.0041 \mathrm{~s}^{-1}$ and $0.0142 \mathrm{~s}^{-1}$ in loaded and unloaded fascicles, respectively, and an associated disruption of connexin 43 gap junction localization within the loaded fascicles, with a clear loss of the discrete staining between tenocytes. The disruption of connexin 43 gap junctions in response to external mechanical stimulation is consistent with previous findings in tenocytes and other cell types (DePaola et al. 1999; Banes et al. 1999a). For example, the application of an intermittent cyclic 5\% tensile strain at $1 \mathrm{~Hz}$ to tenocytes in monolayer culture is reported to cause a reduction in connexin 43 protein expression (Banes et al. 1999a). Similarly, laminar flow shear stress applied to vascular endothelial cells for $5 \mathrm{~h}$ induces a loss of intercellular communication and an associated absence of connexin 43 staining at intercellular regions, although the junctions were reformed and communication re-established after $30 \mathrm{~h}$ (DePaola et al. 1999). The longer term effects of the application of the $1 \mathrm{~N}$ static loading regime on tenocyte metabolism and communication are unknown. Interestingly, loading for $1 \mathrm{~h}$ also induced an increase in Cx43 mRNA expression. In addition, gap junction communication between tenocytes was maintained within the $1 \mathrm{~N}$ loaded samples, albeit at a lower level than for the unloaded samples. The concomitant upregulation of $\mathrm{Cx} 43$ expression may suggest that tenocytes may respond to the reduced communication and disruption of gap junction organization via upregulation of gap junction turnover, associated with enhance connexin synthesis that may result in the subsequent re-establishment of functional gap junctions at later time points. This may, potentially, be associated with the activation of a recovery response 
similar to that reported previously following extended periods of mechanical loading (Maeda et al. 2009).

In summary, we have developed and validated a method to quantify the intercellular gap junction permeability in tendon fascicles using a combined experimental and mathematical approach based upon FLIP. Using this approach, we demonstrate that tensile loading reduces intercellular permeability via gap junction between adjacent tenocytes. This was associated with an increase in connexin 43 mRNA expression, suggesting a complex mechanosensitive breakdown and remodelling of essential gap junction communication. This is therefore the first study to show that tenocyte gap junctions are not only important in transmitting mechanically activated signals but that mechanical loading directly regulates gap junction permeability.

Acknowledgments The present study was funded by an the UK Engineering and Physical Science Research Council (EPSRC) Platform Grant (No. EP/E046975/1).

Open Access This article is distributed under the terms of the Creative Commons Attribution Noncommercial License which permits any noncommercial use, distribution, and reproduction in any medium, provided the original author(s) and source are credited.

\section{References}

Abbaci M, Barberi-Heyob M, Stines JR, Blondel W, Dumas D, Guillemin F, Didelon J (2007) Gap junctional intercellular communication capacity by gap-FRAP technique: a comparative study. Biotechnol J 2:50-61

Abreu E, Leigh D, Derwin K (2008) Effects of altered mechanical load condition on the structure and function of cultured tendon fascicles. J Orthop Res 26:364-373

Arnoczky SP, Tian T, Lavagnino M, Gardner K (2004) Ex vivo static tensile loading inhibits MMP-1 expression in rat tail tendons cells through a cytoskeletally based mechanotransduction mechanism. J Orthop Res 22:328-333

Asundi K, Rempel D (2008) Cyclic loading inhibits expression of MMP-3 but not MMP-1 in an in vitro rabbit flexor tendon model. Clin Biomech (Bristol, Avon) 23:117-121

Banes AJ, Horesovsky G, Tsuzaki M, Boitano S, Lawrence WT, Brown T, Weinhold P, Kenamond C, Benjamin M, Ralphs JR, McNeilly C, Burt J, Miller L (1999a) The connexin 43 gap junction is a mechanosensitive gene in avian flexor tendon cells. In: Caterson B, Archer C, Benjamin M, Ralphs J (eds) The biology of the synovial joint. Harwood Academic Publishers, Netherlands pp 279-299

Banes AJ, Weinhold P, Yang X, Tsuzaki M, Bynum D, Bottlang M, Brown T (1999b) Gap junctions regulate responses of tendon cells ex vivo to mechanical loading. Clin Orthop Relat Res 367S: 356-370

DePaola N, Davies PF Jr, Pritchard WF, Florez L, Harbeck N, Polacek DC (1999) Spatial and temporal regulation of gap junction connexin 43 in vascular endothelial cells exposed to controlled disturbed flows in vitro. Proc Natl Acad Sci USA 96:3154-3159

Eckert R (2006) Gap-junctional single-channel permeability for fluorescent tracers in mammalian cell cultures. Biophys J 91:565-579
Flick J, Davkota A, Tsuzaki M, Almekinders L, Weinhold P (2006) Cyclic loading alters biomechanical properties and secretion of $\mathrm{PGE}_{2}$ and $\mathrm{NO}$ from tendon explants. Clin Biomech (Bristol, Avon) 21:99-106

Garcia M, Knight MM (2010) Cyclic Loading Opens Hemichannels to Release ATP as Part of a Chondrocyte Mechanotransduction Pathway. J Orthop Res 28:510-515

Goldberg GS, Lampe PD, Nicholson BJ (1999) Selective transfer of endogenous metabolites through gap junctions composed of different connexins. Nat Cell Biol 1:457-459

Kannus P (2000) Structure of the tendon connective tissue. Scand J Med Sci Sports 10:312-320

Lavagnino M, Arnoczky SP, Tian T, Vaupel Z (2003) Effect of amplitude and frequency of cyclic tensile strain on the inhibition of MMP-1 mRNA expression in tendon cells: an in vitro study. Connect Tissue Res 44:181-187

Leigh DR, Abreu EL, Derwin KA (2008) Changes in gene expression of individual matrix metalloproteinases differ in response to mechanical unloading of tendon fascicles in explant culture. J Orthop Res 26:1306-1312

Maeda E, Shelton JC, Bader DL, Lee DA (2007) Time dependence of cyclic tensile strain on collagen production in tendon fascicles. Biochem Biophys Res Commun 362:399-404

Maeda E, Shelton JC, Bader DL, Lee DA (2009) Differential regulation of gene expression in isolated tendon fascicles exposed to cyclic tensile strain in vitro. J Appl Physiol 106:506-512

Maeda E, Fleischmann C, Mein CA, Shelton JC, Bader DL, Lee DA (2010) Functional analysis of tenocytes gene expression in tendon fascicles subjected to cyclic tensile strain. Connect Tissue Res 51:434-444

Marsolais D, Duchesne E, Cote CH, Frenette J (2007) Inflammatory cells do not decrease the ultimate tensile strength of intact tendons in vivo and in vitro: protective role of mechanical loading. J Appl Physiol 102:11-17

McNeilly CM, Banes AJ, Benjamin M, Ralphs JR (1996) Tendon cells in vivo form a three dimensional network of cell process linked by gap junctions. J Anat 189:593-600

Pan J, Zhou X, Li W, Novotny JE, Doty SB, Wang L (2009) In situ measurement of transport between subchondral bone and articular cartilage. J Orthop Res 27:1347-1352

Screen HRC, Lee DA, Bader DL, Shelton JC (2004) An investigation into the effects of the hierarchical structure of tendon fascicles on micromechanical properties. Proc Inst Mech Eng H 218H:109-119

Segretain D, Falk MM (2004) Regulation of connexin biosynthesis, assembly, gap junction formation, and removal. Biochim Biophys Acta 1662:3-21

Wagett AD, Benjamin M, Ralphs JR (2006) Connexin 32 and 43 gap junctions differentially modulate tenocyte response to cyclic mechanical load. Eur J Cell Biol 85:1145-1154

Wall ME, Banes AJ (2005) Early responses to mechanical load in tendon: role for calcium signaling, gap junctions and intercellular communication. J Musculoskelet Neuronal Interact 5:70-84

Wang JHC (2006) Mechanobiology of tendon. J Biomech 39:15631582

Yamamoto E, Iwanaga W, Miyazaki H, Hayashi K (2002) Effects of static stress on the mechanical properties of cultures collagen fascicles from the rabbit patellar tendon. J Biomech Eng 124:85-93

Zhu LJ, Altmann S (2005) mRNA and 18S-RNA coapplication-reverse transcription for quantitative gene expression analysis. Anal Biochem 1:102-109 